Alexandra Blank

Politischer Protest und seine Dynamik 
SOZIALWISSENSCHAFT 
Alexandra Blank

\section{Politischer Protest und seine Dynamik}

Die Auswirkungen von Regierungsstrategien

Mit einem Geleitwort von Prof. Dr. Günter Gabisch 
Blank, Alexandra:

Politischer Protest und seine Dynamik : die Auswirkungen von Regierungsstrategien / Alexandra Blank. Mit einem Geleitw. von Günter Gabisch.

- 1. Aufl.. - Wiesbaden : Dt. Univ.-Verl., 2002

(DUV : Sozialwissenschaft)

Zugl.: Göttingen, Univ., Diss., 2001

\section{Auflage April 2002}

Alle Rechte vorbehalten

(C) Deutscher Universitäts-Verlag GmbH, Wiesbaden, 2002

Lektorat: Ute Wrasmann / Britta Göhrisch-Radmacher

Der Deutsche Universitäts-Verlag ist ein Unternehmen der Fachverlagsgruppe BertelsmannSpringer.

www.duv.de

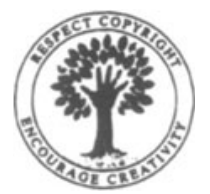

Das Werk einschließlich aller seiner Teile ist urheberrechtlich geschützt. Jede Verwertung außerhalb der engen Grenzen des Urheberrechtsgesetzes ist ohne Zustimmung des Verlags unzulässig und strafbar. Das gilt insbesondere für Vervielfältigungen, Übersetzungen, Mikroverfilmungen und die Einspeicherung und Verarbeitung in elektronischen Systemen.

Die Wiedergabe von Gebrauchsnamen, Handelsnamen, Warenbezeichnungen usw. in diesem Werk berechtigt auch ohne besondere Kennzeichnung nicht zu der Annahme, dass solche Namen im Sinne der Warenzeichen- und Markenschutz-Gesetzgebung als frei zu betrachten wären und daher von jedermann benutzt werden dürften.

Umschlaggestaltung: Regine Zimmer, Dipl.-Designerin, Frankfurt/Main

Gedruckt auf säurefreiem und chlorfrei gebleichtem Papier

ISBN-13: 978-3-8244-4493-9

e-ISBN-13: 978-3-322-85183-3

DOI: 10.1007/ 978-3-322-85183-3 
Für R.B. und L.B. 


\section{Geleitwort}

Das dreitägige Gipfeltreffen der führenden Industrienationen in Genua im Juli 2001 wurde von Krawallen und erbitterten Straßenschlachten zwischen militanten Demonstranten und der Polizei überschattet. Tausende Ordnungskräfte sicherten die Altstadt Genuas; es gab einen Toten und zahlreiche Verletzte; die Kosten der Sicherungsmaßnahmen betrugen schätzungsweise 100 Millionen Euro. Nach den Ausschreitungen von Stockholm ist die "Schlacht von Genua" (Süddeutsche Zeitung vom 21./22.07.2001) trauriger Höhepunkt der Auseinandersetzungen mit der AntiGlobalisierungsbewegung und Beleg für die Relevanz des von der Verfasserin gewählten Themas. Politischer Protest und seine Dynamik ist kein lokales Phänomen sondern ein globales: Globalisierungsgegner sind länderübergreifend organisiert; Proteste finden in verschiedenen Ländern der Welt gleichzeitig statt; die Mobilität der Teilnehmer, unterstützt durch die Informationsmöglichkeiten des Internet, ist enorm. Das Anliegen der Verfasserin besteht darin, mit Hilfe eines formalisierten Kalküls, die Teilnahme des Einzelnen an Protestaktivitäten zu erklären und den Einfluß von Regierungsrepressionen auf den Verlauf von Protestbewegungen aufzuzeigen. Die politischen Ereignisse in den ehemaligen Ostblockstaaten Ende der achtziger Jahre des 20. Jahrhunderts haben gezeigt, daß man nicht in der Lage ist, derartige revolutionäre Entwicklungen in ihrem Ergebnis vorherzusagen. Diese Indeterminiertheit von Protestverläufen modelltheoretisch zu erläutern, ist Hauptanliegen dieser Arbeit.

Die inhaltliche Struktur der Arbeit wird durch drei Schwerpunkte bestimmt:

- Das zweite Kapitel bietet dem Leser eine verständliche und übergreifende Darstellung des konzeptionellen Rahmens der Arbeit. Dieser besteht im wesentlichen aus relevanten Ansätzen der Public-Choice-Theorie, die das Verhalten von Wählern, Regierung und Interessengruppen sowie deren Beziehung untereinander erklärt. Im Zusammenhang mit dem Regierungsverhalten werden zudem neuere Entwicklungen und Modelle aus der Theorie der politischen Konjunkturzyklen aufgegriffen. 
- Das dritte Kapitel beschäftigt sich hauptsächlich mit der Erklärung der politischen Aktivität. Hier wird die Entscheidungssituation des Einzelnen, die Regierungsstrategie und mögliche dynamische Verläufe behandelt.

- Die im zweiten und dritten Kapitel vorgestellten Ansätze werden im folgenden vierten Kapitel zur Formulierung eines eigenen dynamischen Modells benutzt. Während in den diskutierten dynamischen Modellen die angesprochene und aus Erfahrung bestätigte Indeterminiertheit nicht enthalten ist, erlaubt das von der Verfasserin entwickelte Modell chaotische Dynamik und damit den unvorhergesehenen Verlauf und mögliche Eskalation von Protestbewegungen. Die hierzu erforderlichen komplexen formalen Ableitungen werden überwiegend im Anhang behandelt. Illustriert wird diese auf hohem Niveau stattfindende formaltheoretische Analyse durch zahlreiche Beispiele.

Neben einer intensiven Literaturverarbeitung und -aufbereitung zur Thematik des politischen Protests aus ökonomischer Sicht, bietet diese Arbeit gerade in Kapitel 4 eine anspruchsvolle, eigenständige Leistung in der Grundlagenforschung und füllt somit eine Lücke in der Literatur. 


\section{Vorwort}

Die vorliegende Arbeit entstand während meiner Zeit als wissenschaftliche Mitarbeiterin am Volkwirtschaftlichen Seminar der Georg-August-Universität Göttingen. Die Idee zu der doch recht umfangreichen und komplexen Thematik des politischen Protests kam während eines von mir 1997 mit betreuten Seminars über Neue Institutionenökonomik. Genährt und mit neuen Aspekten versorgt wurde dies Vorhaben durch aktuelle Ereignisse wie der Anti-Castor-Demonstrationen und der Ausschreitungen im Rahmen der Anti-Globalisierungsbewegung, die ihren traurigen Höhepunkt im Juli 2001 in Genua fand.

Mein Dank für wertvolle Hinweise, konstruktive Unterstützung und Diskussionsfreudigkeit gilt Herrn PD Dr. Gernot Sieg, Herrn PD Dr. Joachim Ahrens und Herrn Professor Dr. Günter Gabisch. Danken möchte ich Herrn Professor Dr. Hans-Joachim Jarchow für seine Anregungen und für seine Bereitschaft, die Mitbegutachtung zu übernehmen. Für die Durchsicht des Manuskripts geht mein Dank an Frau Niemann, die mehr als einmal dem Fehlerteufel auf die Spur gekommen ist. Nicht vergessen sei das Team am Lehrstuhl VWL II sowie Frau Dr. Dagmar Sakowsky. Auch ihnen gebührt Dank für geleistete Arbeit und Hilfestellungen sowie für das positive Klima in der Zusammenarbeit am Lehrstuhl und am Seminar. Nicht zu unterschätzende moralische und praktische Unterstützung erfuhr ich von meiner Familie, Freunden und Bekannten, insbesondere von Frau Bettina Knop und Frau Tina Leidner sowie von Damian und Frederik Böcker. Ihnen allen gilt mein ausdrücklicher Dank.

Ganz besonders erwähnen und danken möchte ich meinem Mann Ralf Böcker, der mir oft den Rücken freihielt, mich motivierte und immer wieder Geduld und Nervenstärke bewies. Ihm und unserer Tochter Luca Katharina möchte ich dieses Buch widmen. 


\section{Inhaltsverzeichnis}

Abbildungsverzeichnis $\quad$ xiii

Tabellenverzeichnis $\quad$ xv

1 Einleitung 1

1.1 Problemstellung . . . . . . . . . . . . . . . . . . 1

1.2 Theoretische Vorüberlegungen . . . . . . . . . . . . . . . . . . . . . . . . . .

1.3 Aufbau der Arbeit . . . . . . . . . . . . . . . . . . . 18

2 Grundlagen der Public-Choice-Theorie 19

2.1 Wähler und Wahlregeln . . . . . . . . . . . . . . . 21

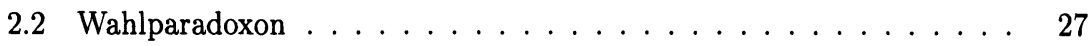

2.3 Verhalten der Regierung . . . . . . . . . . . . . . . . . . 40

2.3.1 Wettbewerb der Parteien um Wählerstimmen . . . . . . . 40

2.3.2 Politische Konjunkturzyklen . . . . . . . . . . . . 45

2.4 Interessengruppen . . . . . . . . . . . . . . . . 59

2.4.1 Mancur Olsons Kollektives Handeln . . . . . . . . . . . 60

2.4.2 Das Chicago-Modell . . . . . . . . . . . . . . . 63

2.4 .3 Rent-Seeking . . . . . . . . . . . . . . . 66

2.4.4 Wettbewerb der Interessengruppen $\ldots \ldots \ldots 69$

2.5 Zusammenfassung . . . . . . . . . . . . . . . 73

3 Erklärung der politischen Aktivität $\quad \mathbf{7 7}$

3.1 Entscheidung des Individuums . . . . . . . . . . . . . . . . . . . . . . 77

3.1.1 Ein Rational-Choice-Modell . . . . . . . . . . . . . . . . 83

3.1 .2 Optimaler Zeitaufwand . . . . . . . . . . . . . 87

3.1.3 Mitwirkung in Interessengruppen . . . . . . . . . . . . . 96

3.2 Strategie der Regierung . . . . . . . . . . . . . . . . . . . 103

3.3 Auswirkungen der Teilnahmeentscheidung . . . . . . . . . . . . 106

3.4 Proteste initiiert von der Oppositionspartei . . . . . . . . . . . . 111 
3.5 Proteste als Signal der Wähler . . . . . . . . . . . . . . . . . . 114

3.6 Proteste als Signal von Interessengruppen _ . . . . . . . . . 125

3.7 Dynamik des Protestverlaufs . . . . . . . . . . . . . . . . . 127

3.8 Zusammenfassung . . . . . . . . . . . . . . . . . . . . . 142

4 Indeterminiertheit des Protestverlaufs 147

4.1 Fragestellung . . . . . . . . . . . . . . . . . . 147

4.2 Verhaltensannahmen . . . . . . . . . . . . . . . 150

4.2 .1 Modellvariablen . . . . . . . . . . . . . . . 154

4.2 .2 Modelleigenschaften . . . . . . . . . . . . 159

4.3 Protestverlauf bei niedrigem Repressionspotential . . . . . . . . . 161

4.3.1 Dynamische Analyse . . . . . . . . . . . . . . . 161

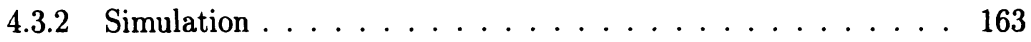

4.4 Protestverlauf bei hohem Repressionspotential . . . . . . . . . . 167

4.4.1 Dynamische Analyse . . . . . . . . . . . . . . 167

4.4 .2 Simulation . . . . . . . . . . . . . . . 178

4.5 Zusammenfassung und Ausblick . . . . . . . . . . . . . . . . 192

$\begin{array}{lll}5 & \text { Schlußbemerkung } & 197\end{array}$

$\begin{array}{ll}\text { A Chaostheoretische Grundlagen } & 201\end{array}$

$\begin{array}{ll}\text { B Beweise } & 225\end{array}$

$\begin{array}{ll}\text { Literaturverzeichnis } & 233\end{array}$

$\begin{array}{ll}\text { Stichwortverzeichnis } & 247\end{array}$ 


\section{Abbildungsverzeichnis}

1.1 Zusammenhang zwischen den Akteuren . . . . . . . . . . 8

2.1 Aufteilung des Individuums in verschiedene Rollen im politischen Prozeß 21

2.2 Wohlfahrtsverluste durch Lobbying nach Olson . . . . . . . . . . 62

2.3 Stimmenmaximierung und Ausmaß der Regulierung . . . . . . . . . 65

2.4 Rent-Seeking . . . . . . . . . . . . . . . . . . 67

2.5 Ausgaben und Steuern bei Stimmenmaximierung. . . . . . . . . . . 68

3.1 Zeitlicher Ablauf des Signalspiels . . . . . . . . . . . . . . 118

4.1 Graphische Darstellung der Aktiven und Passiven. . . . . . . . . . . 158

4.2 Funktion $\Phi_{c, b}$ für $c-b>0 \ldots \ldots \ldots \ldots \ldots$

4.3 Periodic Plot für $c>1, b<1 \ldots \ldots \ldots \ldots \ldots \ldots$

4.4 Periodic Plot für $d \in[0.02,0.12], b \in[0.1,0.9] \ldots \ldots \ldots \ldots \ldots$

4.5 Funktion $\Phi_{c, b}$ und Escape für $c-b<0 \ldots \ldots \ldots \ldots \ldots$

4.6 Graph von $\Phi_{b}$ und stückweise lineare Funktion $L_{b} \ldots \ldots \ldots \ldots$

4.7 Escapemengen für $\Phi_{c, b} \ldots \ldots \ldots \ldots \ldots \ldots \ldots$

4.8 Parameterwerte und Escape für $\Phi_{c, b} \ldots \ldots \ldots \ldots \ldots \ldots \ldots$

4.9 Bifurkationsdiagramm $b \in[2 ; 3,8], c=0,2: \ldots \ldots \ldots \ldots \ldots$

4.10 Bifurkationsdiagramm $b \in[3,4 ; 3,9], c=0,2 \ldots \ldots \ldots \ldots$

4.11 Lyapunov Bifurkation . . . . . . . . . . . . . . . . . . . 180

4.12 Periodic Plot für $b \in[1,4] \ldots \ldots \ldots \ldots \ldots \ldots$

4.13 Periodic Plot für $b \in[3,3.9] . \ldots \ldots \ldots \ldots \ldots \ldots$

4.14 Chaotic Parameter Plot für $b \geq 3,4 \ldots \ldots \ldots$

4.15 Chaotic Parameter Plot für $d$ und $b$ mit $c=0,2 \ldots \ldots \ldots 187$

4.16 Periodic Plot für $d$ und $b$ mit $c=0,2 \ldots \ldots \ldots \ldots 18 \ldots$

4.17 Lyapunov Bifurkation für $d$ mit $c=0,2$ und $b=3,4 \ldots \ldots \ldots$

A.1 Escape . . . . . . . . . . . . . . . . . . . . . . 221

B.1 Funktion $h_{c, b}$ für $c-b<0 \ldots \ldots \ldots \ldots \ldots \ldots \ldots \ldots$ 


\section{Tabellenverzeichnis}

2.1 Auszahlungsmatrix im Minimax-Regret-Spiel . . . . . . . . . . . 29

2.2 Regretmatrix im Minimax-Regret-Spiel . . . . . . . . . . . . 30

2.3 Klassifizierung der Modelle der politischen Konjunkturtheorie . . . . 47

3.1 Teilnahmeentscheidung im Gefangenendilemma . . . . . . . . . 80

3.2 Teilnahmeentscheidung im Assurance Game . . . . . . . . . . . 81

3.3 3-Personen-Gefangenendilemma . . . . . . . . . . . . . 82

3.4 Variablen des Modells von Tullock . . . . . . . . . . . . . 85

3.5 Gewaltanwendung im PR-Game . . . . . . . . . . . . . . . . 104

4.1 Lyapunov-Exponenten . . . . . . . . . . . . . . . 181

B.1 Parameterwerte für Escape . . . . . . . . . . . . . . . . 232 